\title{
Prognostic impact of disseminated tumor cells and microRNA-17-92 cluster deregulation in gastrointestinal cancer
}

\author{
MANUEL VALLADARES-AYERBES ${ }^{1,2}$, MOISES BLANCO ${ }^{2}$, MAR HAZ $^{2}$, VANESSA MEDINA ${ }^{2}$, \\ PILAR IGLESIAS-DÍAZ ${ }^{3}$, MARIA J. LORENZO-PATIÑO ${ }^{3}$, MARGARITA REBOREDO ${ }^{1}$, \\ ISABEL SANTAMARINA ${ }^{2}$, ANGÉLICA FIGUEROA ${ }^{2}$, LUIS M. ANTÓN-APARICIO ${ }^{1,4}$ and LOURDES CALVO ${ }^{1}$ \\ ${ }^{1}$ Medical Oncology, University Hospital; ${ }^{2}$ Biomedical Research Institute INIBIC; ${ }^{3}$ Pathology, \\ University Hospital; ${ }^{4}$ Medicine Department, La Coruña University, UDC, Spain
}

Received March 22, 2011; Accepted May 13, 2011

DOI: 10.3892/ijo.2011.1112

\begin{abstract}
The presence of tumor cells in the bone marrow (BM) could be relevant to identifying high risk of disease progression and death in gastrointestinal cancer. However, the molecular profile associated with disseminated tumor cells (DTCs) homing to the BM has yet to be defined. MicroRNAs (miRNA) play key roles in cellular processes implicated in cancer. Thus, we investigated in 38 patients with colorectal, gastric or pancreatic cancer whether the presence of BM-DTCs is associated with a specific miRNA tumor profile and analyzed their potential prognostic impact. DTCs were detected by immunocytochemistry and anti-cytokeratin antibodies in $42.1 \%$ of the patients. miRNAs were isolated from formalin-fixed, paraffin-embedded tumors. qRT-PCR was used for miRNA profiling. No significant associations were found among DTC detection and miRNA deregulation. Kaplan-Meier curves demonstrated significantly reduced progression-free survival (PFS) and overall survival (OS) in the DTC-positive patients. Although miR-21 was upregulated in $90.6 \%$ of the tumors, no associations with outcomes were found. miR-17 and miR-20a (miRNA-17-92 cluster) were upregulated in 33.3 and $42.4 \%$, respectively. Upregulation of both was correlated and found in 30.3\%. Univariate analysis shows that increasing values for miR-20a were significantly associated with reduced PFS (HR 1.022; $\mathrm{p}=0.016)$ and OS (HR 1.027; $\mathrm{p}=0.003$ ). In multivariate Cox models, DTC positivity (HR 4.07; $\mathrm{p}=0.005$ ) and miR-17 overexpression (HR 2.11; $\mathrm{p}=0.003$ ) were significantly associated with a higher risk of disease progression. The presence of DTCs in the BM (HR 3.98; $\mathrm{p}=0.010$ ) and a miR-17 overexpression (HR 2.62; $\mathrm{p}<0.001$ ) were also associated with a risk of death. Our study suggests that the presence of BM-DTCs and the upregulation of the miR-17-92 cluster in
\end{abstract}

Correspondence to: Dr Manuel Valladares-Ayerbes, Medical Oncology, La Coruña University Hospital, Servicio Galego de Saúde (SERGAS), As Xubias 84, CP. 15006, La Coruña, Spain

E-mail: manuel.valladares.ayerbes@sergas.es

Key words: gastrointestinal cancer, disseminated tumor cells, microRNA, prognostic, real-time PCR tumors are both significant but independent prognostic markers in gastrointestinal cancer patients.

\section{Introduction}

Cancers of the gastrointestinal (GI) tract are a leading cause of cancer-associated morbidity and mortality across the world. The predicted numbers of deaths in 2011 in the European Union (1) due to GI cancer are 162,026 deaths for colorectal cancer, followed by 69,304 deaths for pancreatic cancer and 62,340 deaths for stomach cancer. Although multimodal therapies, including improved local treatments, chemotherapy and molecular-targeted agents, have recently been introduced in the clinical care of GI cancer patients, better staging and prognostic factors to guide treatment decisions are clearly required.

Well-characterized biomarkers are needed to personalize therapy and to predict metastatic progression. Tumor seeding is considered an early event in the process of metastasis formation. Therefore, the detection of these disseminated tumor cells (DTC) in distant organs such as the bone marrow (BM) could be important to identify patients at a high risk of disease progression and death and might indicate the need for further therapeutic approaches (2). Although significant associations with relapse and survival have been reported (3-12), the prognostic relevance of the detection of BM-DTC in GI cancer patients is controversial (13-15). However, the biological characteristics and proliferative potential of this DTC are poorly understood (16). The identification and characterization of molecules that control cancer cell spread in distant organs is critical to our understanding of cancer dissemination. The molecular profile that links the biologic characteristics of primary GI tumors with the presence of epithelial cells homing to bone marrow has yet to be defined.

Tumor progression and metastasis development are complex processes that involve the activation of oncogenes, functional loss of tumor suppressor genes and microRNA (miRNA) deregulation (17). Mature miRNAs are single-stranded, noncoding RNAs that are involved in many biological pathways. miRNA regulation plays key roles in various cellular processes commonly implicated in cancer, such as differentiation, cell growth, angiogenesis, epithelial-to-mesenchymal transition (EMT) and invasion. An increasing number of studies analyzing 
miRNA expression profiles in gastrointestinal tumors and their potential clinical relevance have been reported (18-25).

miR-21 is upregulated in different tumor types, including lung, breast, prostate, pancreatic, and colorectal cancer (17). In colorectal cancer, high expression of miR-21 is correlated with poor survival, poor therapeutic outcome (19), and development of distant metastasis (20). Overexpression of miR-21 has been included in a seven-miRNA signature that was able to predict relapse-free survival and overall survival (OS) in gastric cancer patients (21). In pancreatic cancer, high miR-21 expression is associated with shorter survival (22) and can predict outcomes in resectable patients treated postoperatively (23).

miR-20a and miR-17 belong to the miR-17-92 miRNA cluster, a family of oncogenic miRNAs commonly deregulated in cancer (24). miR-20a and miR-17 are upregulated in colorectal $(18,19,25)$, gastric $(21,25)$, and pancreatic (25) adenocarcinomas. However, miR-17-92 miRNA cluster deregulation in GI cancer and its relationship with prognosis are poorly defined.

In the present study, our aims were to investigate whether the presence of BM micrometastasis, detected by a standardized immunocytochemical method, is associated with a specific miR-21, miR-20a and miR-17 tumor profile, which could serve as a prognostic factor in gastrointestinal cancer patients.

\section{Patients and methods}

Patient data. Consecutive patients with GI cancer from the Medical Oncology Unit at University Hospital in La Coruña (Spain) were prospectively included in the study. Inclusion criteria were as follows: confirmed pathological diagnosis of invasive adenocarcinoma of the GI tract, including colorectal, gastric and pancreatic tumors; stage I-III cancer with no prior systemic therapy for GI cancer; stage IV cancer without previous systemic therapy or with confirmed cancer progression after such treatment; and provision of written informed consent. Exclusion criteria were defined as follows: any other previous malignancy, coagulation disorders, platelet count less than $20.0 \times 10^{9} \mathrm{~L}^{-1}$, and any previous systemic therapy for cancer except stage IV patients with progressive disease confirmed at the time of BM sampling.

The diagnostic work-up included a clinical examination, blood sampling with CA 19.9 and CEA serum determination, an endoscope (when clinically indicated), a chest X-ray and computed tomography (CT) scan of the abdomen and pelvis. Chest CT was performed on the upper digestive tract, rectal tumors and stage IV patients. Patients were followed to observe disease progression, with imaging every 6-12 weeks.

Serum CEA (with an upper limit of normal of $5 \mathrm{ng} / \mathrm{ml}$ ) and CA 19.9 (with an upper limit of normal of $37 \mathrm{U} / \mathrm{ml}$ ) levels were determined using enzyme immunoassay test kits (Advia Centaur, Siemens Healthcare Diagnostics), according to the manufacturer's instructions.

After informed, written consent was obtained from each patient, BM aspiration was performed under local anesthesia, just before systemic treatment for pathological confirmed GI cancer. In patients who first underwent surgery as loco-regional treatment for primary disease, BM aspirate was obtained after the operation. Otherwise, BM samples were obtained before neo-adjuvant chemotherapy or in the presence of active metastatic disease. BM was aspirated from anterior or posterior iliac crest unilaterally. A skin incision was made to avoid contamination with epidermal cells. The study was approved by the Institutional Review Board of the Ethics Committee of Clinical Investigation of Galicia (Spain), and written informed consent was obtained from all patients.

Pathological analysis. Primary tumors, regional lymph nodes and tissues collected during surgery were processed on a routine diagnostic basis. Histological tumor type, depth of tumor invasion and nodal involvement were analyzed, and the disease was staged and graded according to the TNM/UICC system (26). Vascular and perineural invasion were analyzed. When surgery was not performed, pathologic diagnosis was obtained using endoscope- or radiological-guided biopsy.

Residual disease status at the time of BM aspiration was classified as R0 when no residual disease was present after surgery, $\mathrm{R} 1$ when microscopic residual disease was found, and R2 in the presence of macroscopic disease. Patients from whom BM was obtained before the start of neo-adjuvant systemic treatment were categorized as R2.

Preparation of the bone marrow. Unilateral BM aspiration was performed from anterior or posterior iliac crest under local anesthesia and transferred into heparinized tubes. Mononuclear cells (MNCs) were separated by density-gradient centrifugation using Lymphoprep (Nycomed, Oslo, Norway). MNCs were collected from the interphase layer and washed twice in PBS with $10 \%$ FCS. Cytospins were prepared $\left(5 \times 10^{5} \mathrm{MNCs} / \mathrm{slide}\right)$ on polylysine-coated slides in a Hettich cytocentrifuge. The cytospins were air-dried at RT overnight before freezing at $-80^{\circ} \mathrm{C}$ or immunostaining.

Immunocytochemical staining. Immunocytochemistry (ICC) was performed as described previously (27) using the Vectastain ABC-AP kit (Vector), according to the manufacturer's instructions. Slides $\left(5 \times 10^{5} \mathrm{BM}\right.$ MNCs) were incubated with the anti-cytokeratin (CK) monoclonal antibodies AE1/AE3 (Dako). At least two slides were incubated with a negative control antibody of the same immunoglobulin isotype (IgG1). The visualization stage included use of a Vector Red alkaline phosphatase substrate kit. Endogenous alkaline phosphatase activity was inhibited by addition of levamisole. The slides were counterstained with Gill's hematoxylin to visualize nuclear morphology. The slides were manually screened by light microscopy by two pathologists (PID, MLP) with no knowledge about clinical or follow-up data. All of the stained cells were closely evaluated. Categorization of CK-positive cells was performed according to the recommended guidelines (28). The presence of DTC was recorded as positive when at least one stained cell exhibited typical tumor cell morphology or when this immunostained cell lacked hematopoietic characteristics and was not found in negative controls.

microRNA isolation and $q R T-P C R$ in tumor tissue. microRNAenriched total RNA was isolated from formalin-fixed, paraffin-embedded (FFPE) tumor and normal colonic mucosa samples using the Recover All Total Nucleic Acid Isolation Kit for FFPE Tissues (Ambion/Applied Biosystems, USA), following the manufacturer's instructions.

The mirVana qRT-PCR miRNA Detection Kit and the corresponding mirVana qRT-PCR primer sets (Ambion/Applied 
Biosystems, USA) were used to detect and quantify miR-17 and miR-20a. Results were normalized using 5S rRNA and U6 snRNA (mirVana qRT-PCR Primer Sets for Normalization; Ambion/Applied Biosystems).

miR-21 was detected and quantified using a miRCURY LNA First-Strand cDNA Kit, miRCURY LNA SYBR Green Master Mix and specific miRCURY LNA Primer Sets (Exiqon, USA). Mircury LNA Endogenous Control Primer Sets for 5S and U6 (Exiqon) were used as reference genes.

miRNAs were amplified and detected in a LightCycler 480 (Roche, Germany) real-time thermal cycler, using SYBR Green dye. Melting curves were generated using the LightCycler analysis software to determine whether there were spurious amplification products (29).

The relative expression software tool (REST) was used to analyze the relative miRNA expression in each tumor and control sample (non-tumor colonic tissue) and to determine the fold-difference for miR-17, miR-20a and miR-21 (30). The expression levels of target miRNAs were standardized by an index containing 5S rRNA and U6 snRNA. The REST program is based on the correction for exact PCR efficiencies and the mean crossing point deviation between sample group(s) and control group(s). Subsequently, the expression ratio results of the investigated transcripts were tested for significance by a pair-wise fixed reallocation randomization test and plotted using standard error (SE) estimation using a complex Taylor algorithm. miRNA analyses were performed with no knowledge about clinical or follow-up data.

Study design and statistical analysis methods. Differences in the distribution of variables between patient groups according to the presence of DTC in BM were assessed by Pearson's $\chi^{2}$ test, Fisher's exact test or the Kruskal-Wallis test. Non-normality of the distribution of miRNA expression values was confirmed by the Kolmogorov-Smirnov test. Thus, non-parametric statistics (Mann-Whitney and Kruskal-Wallis tests) were used to analyze the potential correlation between miRNA expression and the clinical and pathological features of the study subjects.

Logistic regression analyses were used to assess the effect of tumor miRNA levels on the risk of DTC detection in BM. Odds ratios (OR) and 95\% confidence intervals (CI) were estimated.

Progression-free survival (PFS) was measured as the time between the baseline BM sampling for DTC analysis and the documentation of first tumor progression based on clinical and radiological studies, second tumor or death (events). OS was measured from the time baseline BM was obtained to the date of death from any cause or date of last follow-up. Patients who were alive and progression-free at the time of analysis were censored by using the time between the BM assessment and their most recent follow-up evaluations. The distribution of time-to-event end points, namely PFS and OS, were estimated using the Kaplan-Meier method and compared using the logrank test.

Multivariate survival analyses (PFS and OS) were performed with Cox proportional hazard regression models. We estimated hazard ratios (HRs), 95\% CI and p-values. All statistical tests were two-sided, and p-values $<0.05$ were considered statistically significant. SPSS software (version 16.0) was used for data analysis.
Table I. Patients and clinicopathological characteristics.

\begin{tabular}{lcr}
\hline & $\mathrm{N}$ & $\%$ \\
\hline Age (years) & & \\
$\quad$ Mean (SD, range) & $62.5(7.9,45-76)$ & \\
$<65$ years & 21 & 55.3 \\
$\geq 65$ years & 17 & 44.7 \\
& & \\
Gender & 13 & 34.2 \\
Female & 25 & 65.8 \\
Male & & \\
& & \\
Primary tumor site & 28 & 73.7 \\
Colon or rectum & 7 & 18.4 \\
Stomach & 3 & 7.9 \\
Pancreas & &
\end{tabular}

Stage
I-II
III
IV

15.8

-II

6

23.7

60.5

$\mathrm{pT}$

pT1-pT2 $\quad 5 \quad 13.1$

pT3 $22 \quad 57.9$

pT4 $\quad 8 \quad 21.1$

$\begin{array}{lll}\text { pTx } & 3 & 7.9\end{array}$

$\begin{array}{lll}\text { pNO } & 14 & 36.8\end{array}$

pN1 $12 \quad 31.6$

$\begin{array}{lll}\mathrm{pN} 2 & 7 & 18.4\end{array}$

$\begin{array}{lll}\mathrm{pNx} & 5 & 13.2\end{array}$

M

M0 $15 \quad 39.5$

$\begin{array}{lll}\text { M1 } 23 & 60.5\end{array}$

R status

$\begin{array}{lll}\text { R0-R1 } & 13 & 34.2\end{array}$

$\begin{array}{lll}\mathrm{R} 2 & 25 & 65.8\end{array}$

Location of metastasis

Liver

Extra-hepatic metastasis

Liver and extra-hepatic

$8 \quad 34.8$

$8 \quad 34.8$

$\begin{array}{ll}7 & 30.4\end{array}$

Grade

$\begin{array}{lll}\text { Low grade } & 15 & 39.5\end{array}$

$\begin{array}{lll}\text { High grade } & 23 & 60.5\end{array}$

Vascular/perineural invasion

$\begin{array}{lrr}\text { Yes } & 18 & 47.4 \\ \text { No } & 11 & 28.9 \\ \text { Unknown } & 9 & 23.7\end{array}$

\section{Results}

Patient characteristics. From February 2002 to January 2003, 38 GI cancer patients were included. Clinical characteristics are shown in Table I. Surgery was performed in 30 patients before 
Table II. Distribution of clinicopathological parameters and disseminated tumor cells in bone marrow.

\begin{tabular}{lrrr}
\hline & & \multicolumn{2}{c}{ DTC positive } \\
\cline { 3 - 4 } Parameter & $\mathrm{N}$ & $\%$ & P-value \\
\hline Age (years) & & & 0.917 \\
$\quad<65$ & 9 & 42.9 & \\
$\geq 65$ & 7 & 41.2 & \\
Gender & & & 0.715 \\
$\quad$ Male & 10 & 40 & \\
Female & 6 & 46.1 &
\end{tabular}

Primary tumor site

Colon or rectum

Stomach/pancreas

$10 \quad 35.7$

$6 \quad 60$

Stage

I-II

III

33.3

IV

33.3

$11 \quad 47.8$

pT

pT1-T2

pT3-T4

80

$11 \quad 57.9$

$\mathrm{pN}$

Node negative

Node positive

$8 \quad 57.1$

$6 \quad 31.6$

R status

R0-R1

R2

38.5

$11 \quad 44$

Location of metastasis

None

33.3

Liver

5

46.7

Non-liver metastasis

$4 \quad 50$

Grade

Low grade

High grade

$9 \quad 60$

$\begin{array}{ll}7 & 30.4\end{array}$

Vascular/perineural invasion

No

$5 \quad 45.4$

Yes

38.9

Ca 19.9

$\leq 37$

$>37$

$\begin{array}{ll}10 & 41.7\end{array}$

$5 \quad 38.5$

CEA

$\leq 5$

10

45.4

$>5$

33.3

$0.267^{\mathrm{a}}$

$0.683^{\mathrm{b}}$

$0.141^{\mathrm{a}}$

0.142

0.743

$0.675^{\mathrm{b}}$

0.071

1.000

P-value computed by Pearson's $\chi^{2}$ test. ${ }^{\text {a }}$-value computed by Fisher's exact test. ${ }^{\mathrm{b}} \mathrm{P}$-value computed by Kruskal-Wallis test.

0.461
BM sampling. Median time from surgery to BM aspiration was 7 weeks (mean 26.8; standard error: 7.2; range 3-162 weeks).

$\mathrm{BM}$ aspiration was obtained after R0 or R1 surgery in 13 patients. In this subgroup, $69.2 \%$ of the patients suffered PFS events. In 25 patients, BM samples were obtained before neoadjuvant chemotherapy or in the presence of active metastatic disease, both of which were categorized as R2 at the time of BM aspiration. In this subgroup, PFS events were found in $92 \%$ of patients (Fisher's exact test, $\mathrm{p}=0.154$ ).

All patients were followed up until death or the end of the study. The mean follow-up time was 153 weeks (standard error 21.4; median 108.5 weeks; range 2-388 weeks). Progression events were detected in 32 patients (84.2\%); 27 patients died (71.1\%). The median PFS was 37 weeks (standard error 9.2; 95\% CI: 18.9-55.1). The median global survival was 137 weeks (standard error 44.5; 95\% CI: 49.9-224.1).

Cytokeratin immunocytochemistry. Isolated tumor cells in BM were detected using anti-CK monoclonal antibodies AE1/AE3 and standardized morphological criteria (28). At least $2 \times 10^{6} \mathrm{BM}$ MNC cells were screened per patient. DTC was found in BM in 16 patients (42.1\%, standard error: 0.08$)$. The numbers of tumor cells detected were: 1 (five patients), 2 (seven patients), 3 (two patients), 15 (one patient) and 22 (one patient).

Previous surgery was not related to the presence of DTC in BM (Fisher's exact test, $\mathrm{p}=0.698$ ). To explore the possible influence of the time elapsed from the most recent surgery on the presence of DTC, we analyzed the detection of DTC according to time interval from the operation and BM sampling. The median time from surgery to BM was 26.8 weeks \pm 7.2 weeks (SEM) (median, 7 weeks; range, 3-162). The 25th percentile was 5 weeks. There was no significant difference in DTC detection between time intervals ( $\leq 5$ or $>5$ weeks) from the last surgery (50 and $36.4 \%$, respectively, Fisher's exact test, $\mathrm{p}=0.678$ ).

Bone marrow DTC and correlations with clinicopathology. Clinical and pathologic characteristics of the patients and associations with the detection of DTC in BM are shown in Table II. The detection of DTC was not associated with any of the parameters analyzed.

However, Kaplan-Meier survival analyses demonstrated significantly reduced PFS and OS among the DTC-positive patients (Fig. 1). The median PFS was 40 weeks (95\% CI: 12.467.6) for the patients without DTC versus 29 weeks (95\% CI: $0-60.4)$ for the subgroup positive for DTC in BM (log-rank; $\mathrm{p}=0.026$ ). The median OS was 165 weeks (95\% CI: 93.9-236 weeks) in patients without DTC. In contrast, OS was significantly reduced (median 75 weeks; 95\% CI: 22.8-127.2 weeks) in those patients with CK-positive cells in BM (log-rank test; $\mathrm{p}=0.045$. When only stage I-III patients were considered, the presence of DTC in BM defined a subgroup with a lower median survival (137 weeks vs. not reached; log-rank; $\mathrm{p}=0.038$ ).

miRNA expression in tumors. microRNA-enriched total RNA was retrieved from 33 FFPE-tumor samples for miR-17 and miR-20a analysis and from 32 specimens for miR-21 analysis. Expression of miR-17, miR-20a and miR-21 showed a rightskewed distribution over the patient population (Fig. 2). Mean relative expression levels were 10.1 (SEM 3.4; range 0-89.4) for miR-17, 12.7 (SEM 4.2; range 0-110.3) for miR-20a and 24.3 

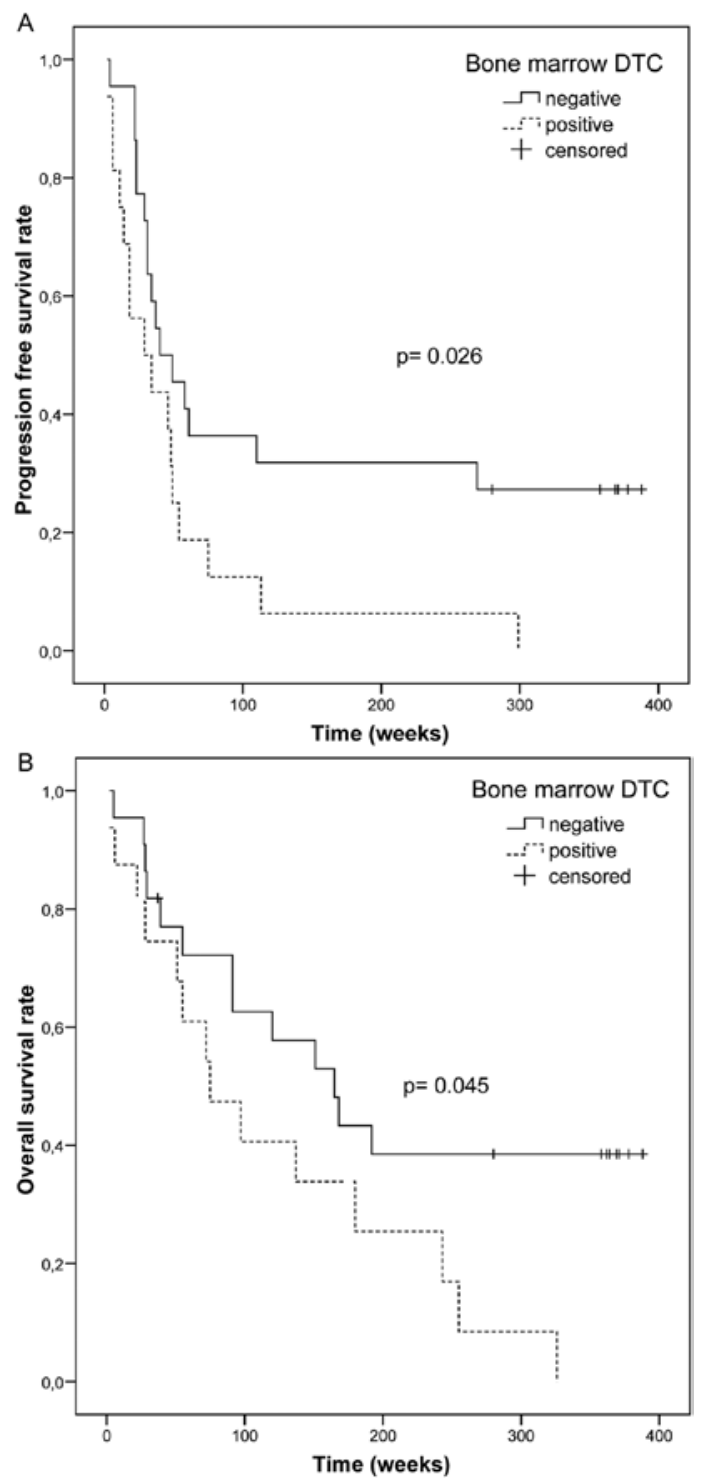

Figure 1. Kaplan-Meier curves depicting progression-free survival (A) and overall survival (B) depending on the detection of disseminated tumor cells in bone marrow. p-values were calculated with the log-rank test.

(SEM 4.6; range 0.5-100.8) for miR-21. In tumors, miR-17 and miR-20a were highly co-expressed (Spearman correlation coefficient $0.650 ; p<0.001)$. No correlations were found between miR-21 and miR-17 ( $\mathrm{p}=0.476)$ or miR-20a $(\mathrm{p}=0.362)$ expression.

Two different strategies were used to categorize relative expression levels for each miRNA in every tumor sample. First, a miRNA was considered up-regulated when the relative expression level was higher than the mean value in the tumor cohort. Using this approach, miR-17 and miR-20a were overexpressed in $27.3 \%$ (9/33) and $21.2 \%$ (7/33) of tumors, respectively. miR-21 was overexpressed in $40.6 \%$ of patients (13/32).

In the second strategy, REST was used as described in Patients and methods (30). The REST output tells the user if the expression ratio results of the investigated targets are up- or down-regulated in the sample group (tumor) in comparison with the control group (non-tumor colonic tissue). Differences in expression between tumor samples and controls were assessed for statistical significance at $\mathrm{p}$-values $\leq 0.01$ by a randomization test. Using REST, miR-21 was up-regulated in $90.6 \%$ (29/32) of the
A

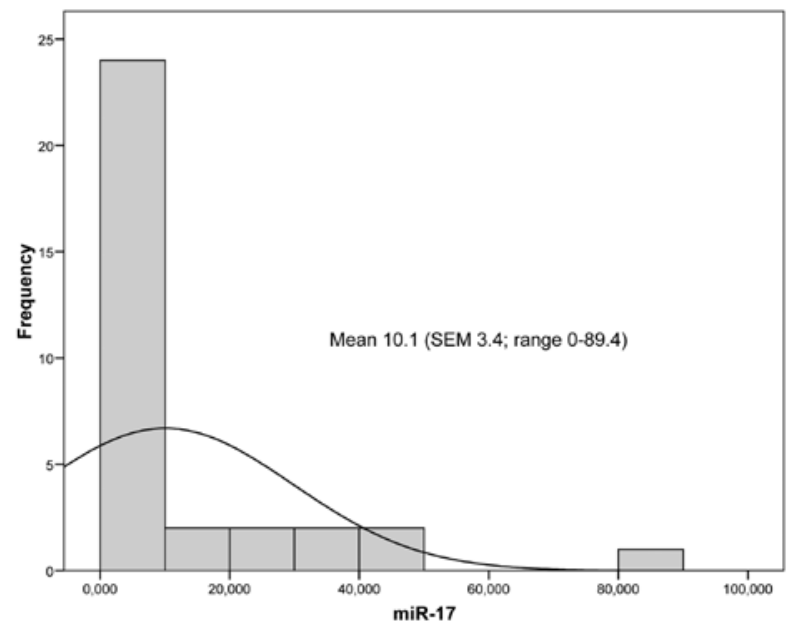

B
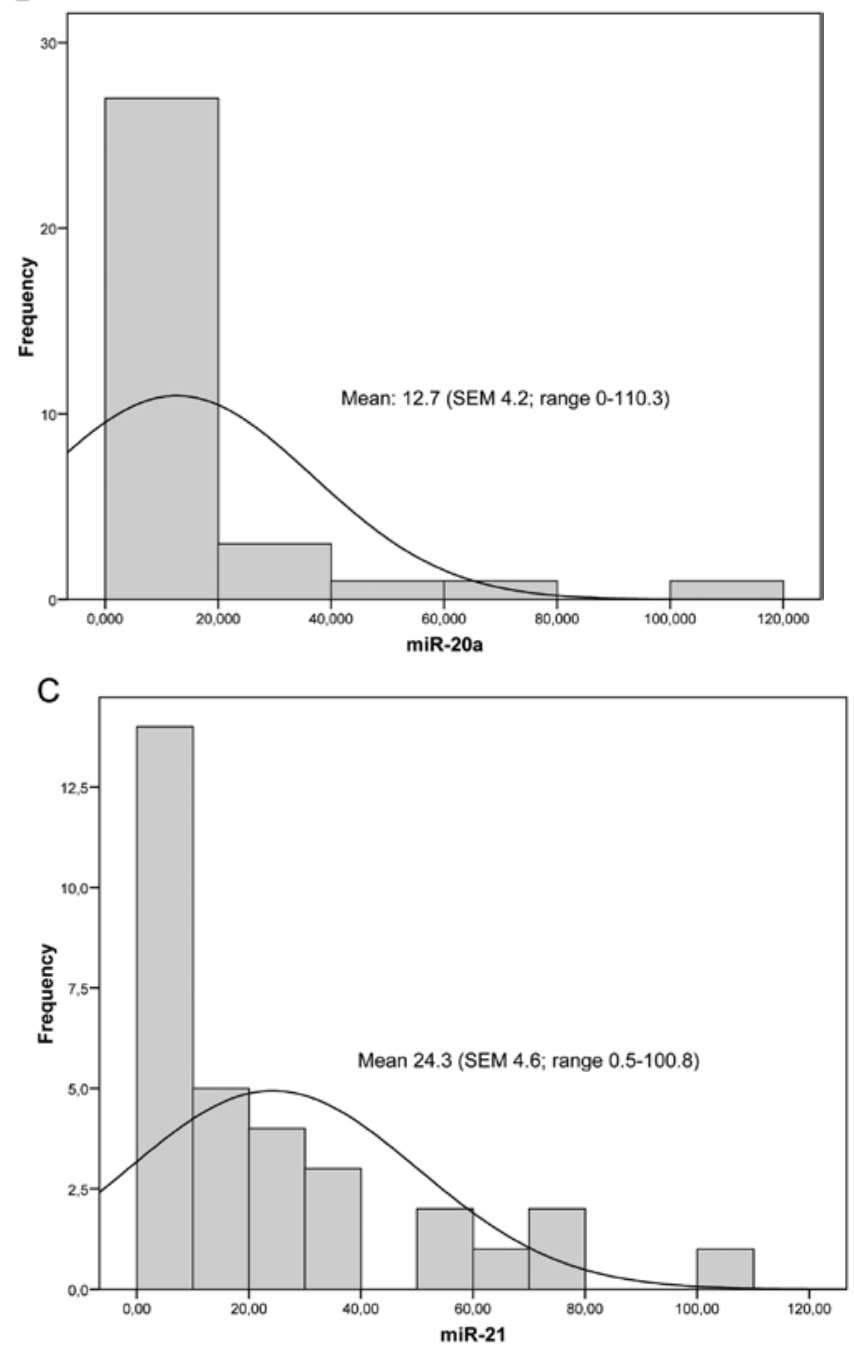

Figure 2. Distribution of (A) miR-17, (B) miR-20a and (C) miR-21 expression in tumors. Frequencies of expression are plotted as a function of the expression values. A continuous line was added to indicate the normal distribution. Mean, SEM and range of miRNA expression values are indicated.

tumors. The REST analysis indicated that miR-17 and miR-20a were up-regulated in $33.3 \%$ (11/33) and $42.4 \%$ (14/33) of tumors, respectively. Up-regulation of both miR-17 and miR-20a was found in 10 patients (30.3\%; Fisher's exact test, $\mathrm{p}<0.001)$. 
Table III. MiRNA expression according to the clinical and pathologic characteristics of the patients.

\begin{tabular}{|c|c|c|c|c|c|c|c|c|c|c|}
\hline & \multirow[b]{2}{*}{$\mathrm{N}$} & \multicolumn{2}{|c|}{ miR-17 } & \multirow[b]{2}{*}{ P-value } & \multicolumn{2}{|c|}{$\operatorname{miR}-20$} & \multirow[b]{2}{*}{ P-value } & \multicolumn{2}{|c|}{ miR-21 } & \multirow[b]{2}{*}{ P-value } \\
\hline & & Mean & SEM & & Mean & SEM & & Mean & SEM & \\
\hline Age (years) & & & & 0.605 & & & 0.334 & & & 0.317 \\
\hline$<65$ years & 19 & 13.69 & 5.59 & & 18.46 & 6.87 & & 28.98 & 7.07 & \\
\hline$\geq 65$ years & 14 & 5.22 & 2.49 & & 4.9 & 2.08 & & 16.93 & 5.15 & \\
\hline Gender & & & & 0.474 & & & 0.837 & & & 0.876 \\
\hline Male & 21 & 10.83 & 4.8 & & 14.92 & 6.32 & & 23.7 & 5.05 & \\
\hline Female & 12 & 8.81 & 4.51 & & 8.84 & 3.22 & & 25.3 & 9.12 & \\
\hline Primary tumor site & & & & 0.983 & & & 0.799 & & & 0.883 \\
\hline Colon or rectum & 23 & 10.54 & 4.25 & & 9.79 & 3.63 & & 23.34 & 5.53 & \\
\hline Stomach/pancreas & 10 & 9.07 & 5.99 & & 19.44 & 11.11 & & 24.22 & 8.53 & \\
\hline Stage & & & & $0.386^{\mathrm{a}}$ & & & $0.963^{\mathrm{a}}$ & & & $0.449^{\mathrm{a}}$ \\
\hline I-II & 6 & 10.87 & 7.21 & & 8.80 & 6.34 & & 13.85 & 6.29 & \\
\hline III & 8 & 4.51 & 3.97 & & 6.80 & 3.41 & & 21.56 & 7.62 & \\
\hline IV & 19 & 12.20 & 5.30 & & 16.43 & 6.82 & & 28.63 & 6.84 & \\
\hline $\mathrm{pT}$ & & & & 0.415 & & & 0.559 & & & 0.686 \\
\hline pT1-pT2 & 5 & 14.69 & 9.03 & & 15.19 & 7.61 & & 38.24 & 19.26 & \\
\hline pT3-pT4 & 25 & 8.44 & 3.85 & & 12.41 & 5.24 & & 23.64 & 4.52 & \\
\hline $\mathrm{pN}$ & & & & 0.459 & & & 0.080 & & & 0.464 \\
\hline Negative & 13 & 3.78 & 2.44 & & 2.78 & 1.47 & & 32.67 & 9.02 & \\
\hline Positive & 15 & 12.21 & 6.25 & & 20.12 & 8.33 & & 24.07 & 5.84 & \\
\hline M & & & & 0.203 & & & 0.827 & & & 0.388 \\
\hline M0 & 14 & 7.23 & 3.76 & & 7.66 & 3.21 & & 17.99 & 4.94 & \\
\hline M1 & 19 & 12.2 & 5.3 & & 16.43 & 6.82 & & 28.63 & 6.84 & \\
\hline Location of metastasis & & & & $0.439^{\mathrm{a}}$ & & & $0.966^{\mathrm{a}}$ & & & $0.474^{\mathrm{a}}$ \\
\hline None & 14 & 7.23 & 3.76 & & 7.66 & 3.21 & & 17.99 & 4.95 & \\
\hline Liver & 11 & 16.46 & 8.81 & & 15.88 & 7.29 & & 34.36 & 10.19 & \\
\hline Non-liver metastasis & 8 & 6.36 & 3.24 & & 17.20 & 13.40 & & 20.74 & 8.16 & \\
\hline R status & & & & 0.052 & & & 0.911 & & & 0.173 \\
\hline R0-R1 & 12 & 3.00 & 2.66 & & 13.65 & 9.1 & & 29.56 & 7.93 & \\
\hline $\mathrm{R} 2$ & 21 & 14.15 & 5,00 & & 12.18 & 4.21 & & 21.15 & 5.58 & \\
\hline Grade & & & & 0.747 & & & 0.311 & & & 0.527 \\
\hline Low grade & 13 & 7.1 & 3.21 & & 5.70 & 2.36 & & 19.75 & 6.42 & \\
\hline High grade & 20 & 12.03 & 5.27 & & 17.27 & 6.59 & & 27.69 & 6.35 & \\
\hline Vascular/perineural invasion & & & & 0.845 & & & 0.429 & & & 0.38 \\
\hline No & 10 & 6.51 & 3.75 & & 17.3 & 10.71 & & 27.38 & 10.12 & \\
\hline Yes & 14 & 10.28 & 6.57 & & 10.18 & 5.76 & & 31.70 & 6.66 & \\
\hline \multicolumn{11}{|l|}{ Serum tumor markers } \\
\hline Ca $19.9>37$ & 13 & 11.56 & 5.26 & 0.116 & 18.97 & 10.01 & 0.487 & 12.18 & 3.22 & 0.071 \\
\hline $\mathrm{CEA}>5$ & 15 & 21.82 & 8.67 & 0.017 & 28.95 & 10.85 & 0.137 & 19.58 & 7.45 & 0.155 \\
\hline
\end{tabular}

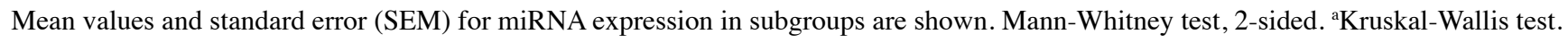



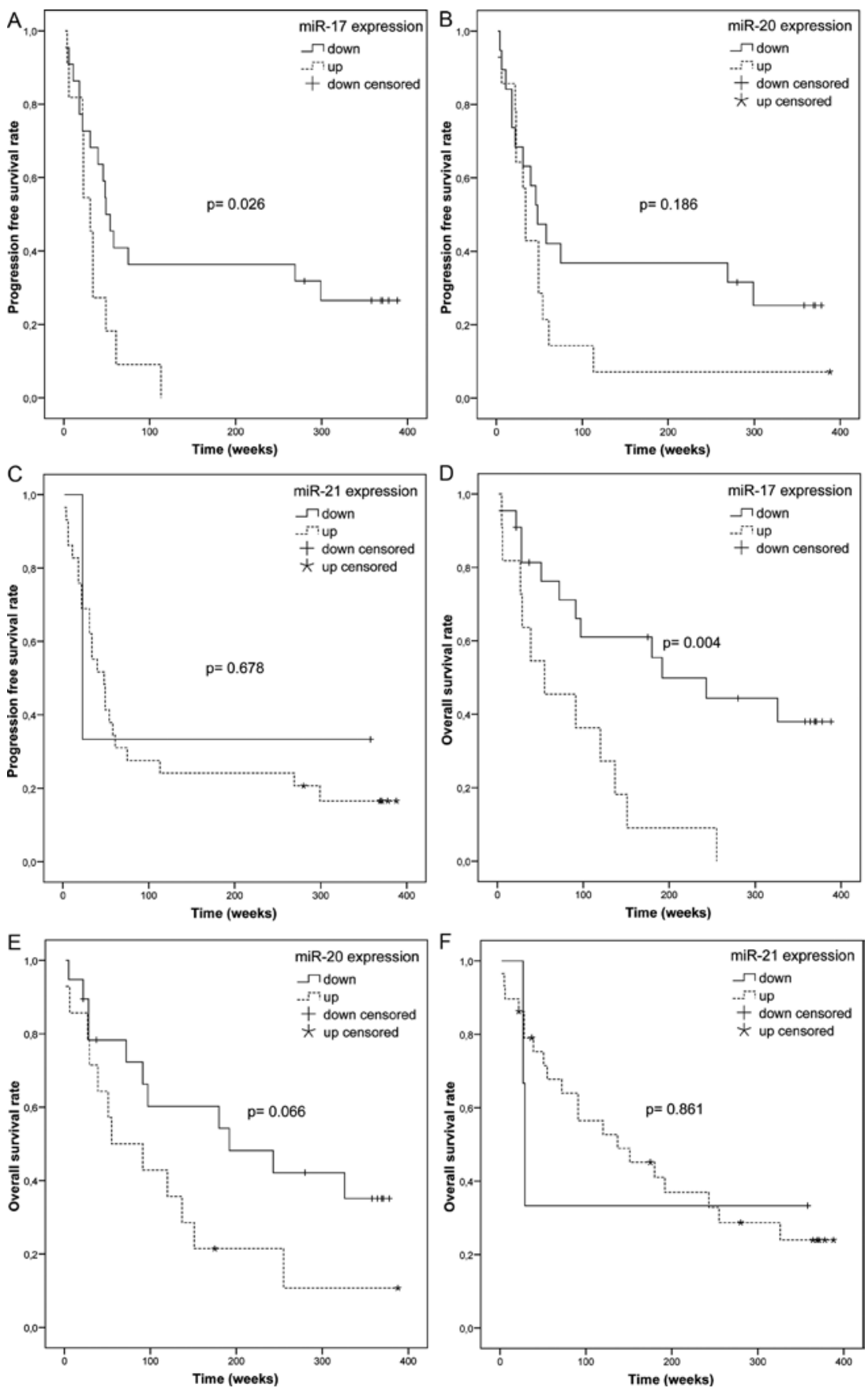

Figure 3. Kaplan-Meier curves depicting progression-free survival (PFS) and overall survival (OS) depending on the tumor miRNA expression. PFS according miR-17 expression (A). PFS according miR-20a expression (B). PFS according miR-21 expression (C). OS according miR-17 expression (D). OS according miR-20a expression (E). OS according miR-21 expression (F). p-values were calculated with the log-rank test.

Associations of deregulated miRNAs with clinical outcome. miRNA expression according to clinical and pathologic characteristics is listed in Table III. Only increased serum CEA level was associated with high miR-17 tumor expression $(\mathrm{p}=0.017)$. Otherwise, no significant associations were found among miRNA tumor expression and any of the clinical or pathologic parameters analyzed. A trend was observed between high expression of miR-17 and macroscopic residual tumor (R2) at the time of BM sampling $(\mathrm{p}=0.052)$, suggesting a more aggressive clinical presentation. Also, a trend was observed between miR-20a expression and lymph node metastasis $(\mathrm{p}=0.08)$. Likewise, a trend between miR-21 tumor expression and serum CA 19.9 level was found $(\mathrm{p}=0.071)$.
To verify the association between miRNA expression and survival (PFS and OS), the miRNA expression data were dichotomized into clearly defined high and low expression groups. This cutoff was set based on REST as described above. Mean values (with SEM) in the low and high expression groups, respectively, for each miRNA were as follows: $0.09(0.05)$ and 30.1 (7.24) for miR-17 ( $<<0.001) ; 0.37$ (0.33) and 29.45 (7.97) for miR-20a $(\mathrm{p}<0.001) ; 0.8(0.23)$ and $26.74(4.82)$ for miR-21 $(\mathrm{p}=0.005)$.

Kaplan-Meier curves for patients categorized according to miRNA expression are shown in Fig. 3. The median PFS for the group with high miR-17 expression and the other group without miR-17 up-regulation were 31 weeks and 49 weeks, respectively (Fig. 3A; $p=0.026$ ). In addition, the differences in median OS 
Table IV. Relations of disseminated tumor cells in bone marrow with tumor miRNAs expression: logistic regression analysis.

\begin{tabular}{|c|c|c|c|c|c|c|c|c|c|c|}
\hline \multirow[b]{2}{*}{ Groups } & \multirow[b]{2}{*}{$\begin{array}{c}\text { DTC positive } \\
\mathrm{N}(\%)\end{array}$} & \multicolumn{3}{|c|}{$\operatorname{mir}-17$} & \multicolumn{3}{|c|}{ miR-20 } & \multicolumn{3}{|c|}{ miR-21 } \\
\hline & & OR & $95 \% \mathrm{CI}$ & P-value & OR & $95 \% \mathrm{CI}$ & $\mathrm{P}$-value & OR & $95 \% \mathrm{CI}$ & P-value \\
\hline Global & $16(42.1)$ & 0.947 & 0.8791 .020 & 0.148 & 1.026 & 0.9611 .095 & 0.438 & 0.998 & 0.9761 .020 & 0.832 \\
\hline \multicolumn{11}{|l|}{ Stage } \\
\hline I-III & $5(33.3)$ & 1.042 & 0.8471 .282 & 0.700 & 0.970 & 0.7691 .224 & 0.800 & 0.943 & 0.8741 .019 & 0.137 \\
\hline IV & $11(47.8)$ & 0.848 & 0.6801 .058 & 0.143 & 1.035 & 0.9031 .187 & 0.618 & 1.020 & 0.9831 .058 & 0.289 \\
\hline \multicolumn{11}{|l|}{ Lymph node } \\
\hline Negative & $8(57.1)$ & 0.218 & 0.0133 .567 & 0.285 & 16.961 & 0.0903202 .2 & 0.290 & 1.022 & 0.9801 .066 & 0.315 \\
\hline Positive & $6(31.6)$ & 0.962 & 0.7971 .160 & 0.681 & 1.117 & 0.8761 .425 & 0.373 & 0.874 & 0.7291 .049 & 0.148 \\
\hline \multicolumn{11}{|l|}{ Primary tumor site } \\
\hline Colon or rectum & $10(35.7)$ & 0.996 & 0.8841 .122 & 0.944 & 0.981 & 0.8471 .136 & 0.798 & 0.997 & 0.9671 .028 & 0.849 \\
\hline Stomach/pancreas & $6(60)$ & 0.711 & 0.3081 .642 & 0.424 & 1.285 & 0.5882 .809 & 0.529 & 1.003 & 0.9641 .044 & 0.870 \\
\hline
\end{tabular}

Table V. Multivariate Cox proportional hazard regression analyses for progression-free survival and overall survival.

\begin{tabular}{|c|c|c|c|c|c|c|c|}
\hline \multirow[b]{2}{*}{ Variable } & \multirow[b]{2}{*}{ Subset } & \multicolumn{3}{|c|}{ Progression-free survival } & \multicolumn{3}{|c|}{ Global survival } \\
\hline & & Hazard ratio & $(95 \% \mathrm{CI})$ & P-value & Hazard ratio & $(95 \% \mathrm{CI})$ & P-value \\
\hline Disseminated tumor cells & Negative/positive & 4.07 & 1.5410 .73 & 0.005 & 3.98 & 1.3911 .41 & 0.010 \\
\hline miR-17 expression & Down-/up-regulated & 2.11 & $1.29 \quad 3.45$ & 0.003 & 2.62 & $1.55 \quad 4.49$ & 0.000 \\
\hline Stage & I-III/IV & 5.30 & 1.5917 .70 & 0.007 & 3.97 & 1.0814 .56 & 0.037 \\
\hline Surgical resection & $\mathrm{R} 0-1 / \mathrm{R} 2$ & 1.10 & $0.60 \quad 2.00$ & 0.766 & 1.15 & $0.50 \quad 2.21$ & 0.681 \\
\hline Primary tumor & Colorectal/non-colorectal & 0.16 & $0.06 \quad 0.45$ & 0.000 & 0.17 & $0.06 \quad 0.53$ & 0.002 \\
\hline
\end{tabular}

according to miR-17 values (55 weeks for the up-regulated group and 192 weeks for the low miR-17 group) were also significant (Fig. 3D; p=0.004). However, Kaplan-Meier estimates of PFS and OS were not significantly correlated with up- or downregulation of miR-20a and miR-21 in tumors (Fig. 3).

HRs for PFS and OS were estimated considering the actual values of every miRNA as a continuous variable in the Cox regression model. Across the entire cohort, increasing values for miR-20a were associated with PFS events (HR 1.022; 95\% CI: $1.004-1.040 ; \mathrm{p}=0.016)$ and reduced OS (HR 1.027; 95\% CI: 1.009-1.046; $\mathrm{p}=0.003)$. When adjusting for TNM/UICC stage as covariate in the Cox model, increasing values for miR-20a and miR-17 were associated with the risk of progression and death in stage I-III patients. The estimated HRs for miR-20a levels were 1.063 (95\% CI, 1.002-1.127; p=0.043) and 1.065 (95\% CI, 1.003-1.130; $\mathrm{p}=0.040$ ) for PFS and OS, respectively. For miR-17, increased values were also significantly associated with risk of progression (HR 1.056; 95\% CI, 1.007-1.107; $\mathrm{p}=0.024)$. The estimated HR for miR-17 values and OS was 1.065 (95\% CI, $0.999-1.102 ; \mathrm{p}=0.052$ ).

No significant associations were found between actual miR-21 values for the entire cohort and PFS (HR 1.007; 95\%
CI, 0.993-1.022; $\mathrm{p}=0.346$ ) or OS (HR 1.0; 95\% CI, 0.983-1.017; $\mathrm{p}=0.988$ ). Moreover, in stage I-III patients, hazard ratios for increasing miR-21 values and PFS (HR 1.005; 95\% CI, 0.966$1.045 ; \mathrm{p}=0.821$ ) or OS (HR 0.985; 95\% CI, 0.939-1.032; $\mathrm{p}=0.52$ ) were not significant.

miRNA deregulation in tumors and bone marrow DTC. We analyzed whether the tumor expression levels of miR-17, miR20a and miR-21 were associated with the presence of DTC in BM. In DTC-positive patients, the mean miR-17 value in tumors was 4.89 (SEM 2.8), which was not significantly different $(p=0.43)$ from those who were DTC-negative (13.9; SEM 5.5). The mean value of miR-20a in tumors from the patients positive for DTC in BM was 13.2 (SEM 7.8) and 12.4 (SEM 4.6) in those patients without DTC $(\mathrm{p}=0.91)$. There was no significant difference $(\mathrm{p}=0.92)$ in mean miR-21 value between DTC-positive (26.4; SEM 8.8) and DTC-negative patients (22.9; SEM 4.97).

Furthermore, to assess independent relationships of DTC in $\mathrm{BM}$ with tumor miRNA expression, logistic regression analysis was performed. OR was adjusted for other covariables, including primary tumor site (colorectal vs. non-colorectal), stage (I-III vs. IV) and lymph node metastasis (negative vs. positive). However, 
the presence of DTC in BM was, again, not associated with miRNA expression (Table IV).

Prognostic significance of DTC and miRNA expression: Cox models. We used multivariate Cox regression analysis to investigate whether the presence of DTC and miRNA expression was an independent predictor of progression-free survival and overall survival in gastrointestinal cancer patients. The stage, extent of surgical resection and location of the primary tumor were used as covariates (Table V).

According to Cox multivariate regression analysis, the factors associated with a higher risk of PFS events were DTC positivity (HR 4.07; $\mathrm{p}=0.005$ ), miR-17 overexpression (HR 2.11; $\mathrm{p}=0.003$ ) and stage IV cancer (HR 5.3; $\mathrm{p}=0.007$ ). The risk of progression was lower in colorectal cancer patients (HR $0.16 ; \mathrm{p}<0.001)$.

The presence of DTC in BM was also associated with poor overall survival (HR 3.98; $\mathrm{p}=0.010$ ) in the multivariate model. Moreover, the higher expression of miR-17 resulted in a 2.62fold increase in the risk of death $(\mathrm{p}<0.001)$. In addition, stage IV (HR 3.97; $p=0.037$ ) was also associated with poor OS. The risk of death was lower in colorectal cancer patients (HR 0.17; $\mathrm{p}=0.002$ ).

\section{Discussion}

In our study, we examined the presence of DTCs in the BM of patients with colorectal, gastric and pancreatic cancer and their clinical outcomes. We then investigated the existence of any correlation between these findings and the tumor expression of selected miRNA. We found that CK-positive bone marrow cells and upregulation of the miR-17-92 microRNA cluster in the tumor were both significant prognostic markers in gastrointestinal cancer patients. Nevertheless, the tumor expression of miR-17, miR-20a and miR-21 was not associated with the presence of disseminated CK-positive cells in the BM.

Although the prognostic relevance of disseminated tumor cells in bone marrow is widely accepted in breast cancer (31), the clinical and biological significance of finding such micrometastasis in GI cancer patients remains controversial. However, the clinical value as a prognostic factor of DTC detection in BM using CK-based immunocytochemistry in GI cancer patients has been suggested in previous studies analyzing colorectal $(4,10)$ and gastric cancer $(7,8)$.

Independent prognostic values for progression-free survival and global survival were found in our study of DTC detection using Kaplan-Meier estimates and multivariate analysis. Furthermore, the presence of DTC in BM defines a subgroup of stage I-III patients with a significantly lower median overall survival.

Although the inclusion of patients with different primary GI tumors and stages could be considered a limitation of this study, we suggest that this pragmatic design accurately reflects the patients that attend the oncology clinic. Thus, the prognostic value of DTC detection and miRNA quantification has been estimated in a cohort of patients truly representative of those found in the clinical setting. In fact, there were no significant differences among the miRNAs relative expressions levels according to location of primary tumor (colorectal or non-colorectal cancer). Furthermore, prognostic impact of DTC detection and
miRNA quantification remain significant in multivariate model adjusting for primary tumor site.

In different studies that used immunocytochemistry, CK-positive cells were detected in the BM of $16-64 \%$ of colorectal (13-15), 25-66\% of gastric $(5-8,13)$ and $21-61 \%$ of pancreatic cancer patients $(9,32)$. In the present report, DTC were found in $42.1 \%$ of patients (35.7\% of colorectal and $64.3 \%$ of gastric and pancreatic cancer patients). Interestingly, and in agreement with the results of previous publications (13), the detection of DTC was not associated in our study with TNM/ UICC stage, pathological grade, vascular or perineural invasion or the extent of surgical resection. Therefore, we hypothesized that the presence of cancer cells in the bone marrow may reflect the distinct biological properties of a tumor. These unique biological characteristics of the primary tumors may influence the ability of cancer cells to disseminate in certain distant organs and subsequently trigger metastasis formation. Thus, combined analyses of the primary tumor histopathology, as well as their genetic and transcriptomic backgrounds and DTC status, will increase our understanding of invasion and overt metastasis development in GI cancer.

At present, little is known about the characteristics of primary gastrointestinal tumors that might have a role in the early shedding of tumor cells into the bloodstream and subsequent homing to bone marrow. The presence of disseminated carcinoma cells in bone marrow in patients with various types of epithelial tumors, including colorectal, gastric and pancreatic cancer, is not associated with TP53 gene mutations in the primary tumor (33). Markers of tumor angiogenesis, such as microvessel counts and VEGF-A expression in primary gastric cancer, have been correlated with the presence of DTC in bone marrow $(34,35)$. A significant association between CXCR4-positive expression of cancer cells in the primary tumor and the presence of CK-positive cells in the bone marrow has been described in patients with squamous cell carcinoma or adenocarcinoma of the esophagus (36). Tumor expression of the neural cell adhesion molecule L1 (CD171) is associated with micrometastatic spread and poor outcome in colorectal cancer (37).

Recently, attention has focused on the role of miRNA regulation in essential mechanisms for cancer progression and metastasis, including invasion, proliferation, cell migration, EMT, angiogenesis and apoptosis $(17,18,24)$. Therefore, we investigated whether the occurrence of DTC in bone marrow was associated with miRNA tumor expression and the related potential prognostic value. Furthermore, the stability of miRNAs in formalin-fixed, paraffin-embedded tissue and the suitability for real-time PCR-based assays, as a gold-standard method for quantification, are clearly advantageous for biomarker research (38).

Here, tumor overexpression of the miR-17-92 cluster emerged as a compelling prognostic indicator in GI cancer. This was independently confirmed by a multivariate Cox proportional hazard model, which defined miR-17 upregulation as a statistically significant independent predictor of poor PFS and OS. Furthermore, Cox regression models showed that increasing continuous values for miR-20a and miR-17 were both associated with the risk of progression and death in stage I-III patients.

Nonetheless, deregulated expression of the miRNAs was not associated with any of the clinicopathological tumor parameters investigated or with the detection of CK-positive 
cells in the BM. A significant association was observed only between high expression of miR-17 and CEA level.

Preclinical and translational studies have established the oncogenic role of the miR-17-92 cluster in several hematological and solid tumors $(24,25)$. All six members of the mir-17-92 polycistron on chromosome 13 (miR-17, miR-18a, miR-19a, miR-20a, miR-19b-1 and miR-92a-1) are some of the highestoverexpressed miRNAs in several cancer types $(25,29)$.

It appears reasonable to assume that the prognostic impact of the up-regulation of miR-17 and miR-20a in GI cancer is attributable in part to the mechanistic relevance of their target genes (40). mir-17 and miR-20a down-regulate the activating members of the $E 2 F$ family of transcription factors $(E 2 F 1, E 2 F 2$, and $E 2 F 3)$ and the cyclin-dependent kinase inhibitor CDKN1a/p21, which are regulators of the G1-to-S-phase transition in the cell cycle. Furthermore, the apoptosis inducer BIM and the proto-oncogene $L R F$, implicated in senescence, are also under the control of the miR-17-92 cluster (40). A recent report has identified hypoxiainducible factor (HIF)-1A as a novel direct target of miR-17-5p and miR-20a (41). Furthermore, overexpression of the miR-17-92 cluster markedly inhibits hypoxia-induced apoptosis in colon cancer cell lines through the regulation of p53-mediated transcriptional repression (42). Recent data suggest that miR-17-5p and miR-20a directly control the expression of the type II TGF $\beta$ receptor in colorectal cancer progression, inhibiting the transcription of individual TGF $\beta$-responsive genes and indirectly stimulating angiogenesis through inhibition of a wide repertoire of anti-angiogenic factors (43).

Nevertheless, studies on miR-17-92 cluster deregulation in GI cancer in the clinical setting and its potential prognostic impact are limited. Altered expression of miR-17-5p is associated (44) with vascular invasion and $\mathrm{LOH}$ in the TP53 region in a series of colorectal cancer patients. A trend between poor disease-free survival and upregulation of miR-17-5p has also been observed, but only in stage I-II patients. In that series, downregulation of its paralog miR-106a was independently associated with DFS and OS (44). Contradictory results in relation to miR-106a have been reported elsewhere (19). In that study, in an analysis designed to identify whether any of the overexpressed miRNAs in colorectal cancer identified by microarray experiments are associated with poor survival, up-regulation of miR-20a, miR-21, miR-106a, miR-181b and miR-203 was significant in the training cohort. However, only miR-21 retained the prognostic impact in the validation cohort. Tumors with high expression of miR-21 were associated with poor survival outcome and poor response to adjuvant chemotherapy independent of staging and other clinical covariates (19). Likewise, in gastric cancer patients, miR-21 was overexpressed and selected in the progression signature but not in the survival signature (45).

In our study, increased miR-21 level was found in $90.6 \%$ of tumors, consistently with previous studies investigating patients with colon $(25,27,39)$, gastric $(21,39,45,46)$ and pancreatic $(22,23,29)$ adenocarcinomas. However, we did not find any significant associations between miR-21 level and the clinical and pathologic characteristics, in line with recently reported results (47). Likewise, in our series, expression of miR-21 was not associated with any of the clinical outcomes analyzed.

miR-21, acting as an oncogene (48), targets the products of several genes relevant to cancer progression and metastasis, including PDCD4, SPRY, PTEN and TPM1. Recent data suggest that miR-21 induces a paradoxical negative regulation of cell cycle progression in hypoxic colon cancer models, through a Cdc25a protein phosphatase-dependent mechanism (49). Interestingly, in situ hybridization results indicate that miR-21 is located primarily in the stromal compartment of the tumors (50). These data suggest a set of connections between tumor cells and their microenvironment, where the net effect of a particular miRNA might be variable, in a cellular context-dependent manner.

Furthermore, technical differences could explain apparently contrasting results. In fact, in the study reported by Schepeler et al (51), there was no correlation between miR-21 expressions data obtained with real-time RT-PCR as compared to data obtained with microarray with probes designed against the mature form. Also, the limited numbers of patients included in the studies, including ours, make the data more susceptible to stochastic effects.

When we considered the different reports about the potential prognostic relevance of miRNA expression in GI cancer, a considerable degree of inter-study heterogeneity was noted. Differences in the detection and quantification methods (microarrays, qRT-PCR), types and numbers of miRNAs evaluated (pre-miRNA or mature form, expression profile, single marker), and sample source (FFPE, deep-frozen, specimen microdissection), as well as in the clinicopathological data of the included patients, ought to be considered as potential sources of heterogeneity.

In conclusion, our study suggests that the presence of CK-positive cells in the bone marrow and the upregulation of the miR-17-92 cluster in the tumor were both significant but independent prognostic markers in gastrointestinal cancer patients. These results will require validation in independent and large sample sets before firm conclusions can be reached.

\section{Acknowledgments}

The authors thank the patients for their participation in the study. The excellent collaboration of the Oncology staff nurses is also recognized. This study was supported by grants PI061541 (Fondo de Investigaciones Sanitarias, Instituto Carlos III), PS 08/77 and PGIDT01PXI90001PR (Servicio Galego de Saúde and Xunta de Galicia). M. Blanco and M. Haz are supported in part by a research contract with Fondo de Investigaciones Sanitarias, Instituto Carlos III (Spain). A. Figueroa is supported by 'Isidro Parga Pondal' research contracts with Xunta de Galicia (Spain). Cancer research in our laboratory is supported by the -Fundación Complejo Hospitalario Universitario La Coruña'

\section{References}

1. Malvezzi M, Arfé A, Bertuccio P,Levi F, La Vecchia C and Negri E: European cancer mortality predictions for the year 2011. Ann Oncol. Advance Access published February 8, 2011. doi:10.1093/ annonc/mdq774

2. Pantel K, Alix-Panabières $\mathrm{C}$ and Riethdorf S: Cancer micrometastases. Nat Rev Clin Oncol 6: 339-351, 2009.

3. Schlimok G, Funke I, Bock B, Schweiberer B, Witte J and Riethmüller G: Epithelial tumor cells in bone marrow of patients with colorectal cancer: immunocytochemical detection, phenotypic characterization, and prognostic significance. J Clin Oncol 8: 831-837, 1990.

4. Lindemann F, Schlimok G, Dirschedl P, Witte J and Riethmüller G: Prognostic significance of micrometastatic tumour cells in bone marrow of colorectal cancer patients. Lancet 340: 685-689, 1992. 
5. Heiss MM, Allgayer H, Gruetzner KU, et al: Individual development and uPA-receptor expression of disseminated tumour cells in bone marrow: a reference to early systemic disease in solid cancer. Nat Med 1: 1035-1039, 1995

6. Maehara Y, Yamamoto M, Oda S, Baba H, Kusumoto T and Ohno S: Cytokeratin-positive cells in bone marrow for identifying distant micrometastasis of gastric cancer. Br J Cancer 73: 83-87, 1996.

7. Jauch KW, Heiss MM, Gruetzner U, Funke I, Pantel K, Babic R, Eissner HJ, Riethmueller G and Schildberg FW: Prognostic significance of bone marrow micrometastases in patients with gastric cancer. J Clin Oncol 14: 1810-1817, 1996.

8. Schott A, Vogel I, Krueger U, Kalthoff H, Schreiber HW, Schmiegel W, Henne-Bruns D, Kremer B and Juhl H: Isolated tumor cells are frequently detectable in the peritoneal cavity of gastric and colorectal cancer patients and serve as a new prognostic marker. Ann Surg 227: 372-379, 1998.

9. Roder JD, Thorban S, Pantel K and Siewert JR: Micrometastases in bone marrow: prognostic indicators for pancreatic cancer World J Surg 23: 888-891, 1999.

10. Leinung S, Würl P, Schönfelder A, Weiss CL, Röder I and Schönfelder M: Detection of cytokeratin-positive cells in bone marrow in breast cancer and colorectal carcinoma in comparison with other factors of prognosis. J Hematother Stem Cell Res 9: 905-911, 2000

11. Z'graggen K, Centeno BA, Fernandez-del Castillo F, Jimenez RE, Werner J and Warshaw AL: Biological implications of tumor cells in blood and bone marrow of pancreatic cancer patients. Surgery 129: 537-546, 2001.

12. Gretschel S, Schick C, Schneider U, Estevez-Schwarz L, Bembenek A and Schlag PM: Prognostic value of cytokeratinpositive bone marrow cells of gastric cancer patients. Ann Surg Oncol 14: 373-380, 2007

13. Funke I and Schraut W: Meta-analyses of studies on bone marrow micrometastases: an independent prognostic impact remains to be substantiated. J Clin Oncol 16: 557-566, 1998.

14. Rahbari NN, Aigner M, Thorlund K, Mollberg N, Motschall E, Jensen K, Diener MK, Büchler MW, Koch M and Weitz J: Meta-analysis shows that detection of circulating tumor cells indicates poor prognosis in patients with colorectal cancer. Gastroenterology 138: 1714-1726, 2010.

15. Steinert R, Hantschick M, Vieth M, Gastinger I, Kühnel F, Lippert $\mathrm{H}$ and Reymond MA: Influence of subclinical tumor spreading on survival after curative surgery for colorectal cancer. Arch Surg 143: 122-128, 2008

16. Solakoglu O, Maierhofer C, Lahr G, Breit E, Scheunemann P, Heumos I, Pichlmeier U, Schlimok G, Oberneder R, Kollermann MW, Kollermann J, Speicher MR and Pantel K: Heterogeneous proliferative potential of occult metastatic cells in bone marrow of patients with solid epithelial tumors. Proc Nat Acad Sci USA 99: 2246-2251, 2002.

17. Croce CM: Oncogenes and cancer. N Engl J Med 358: 502-511, 2008

18. Motoyama K, Inoue H, Takatsuno Y, Tanaka F, Mimori K, Uetake H, Sugihara K and Mori M: Over- and under-expressec microRNAs in human colorectal cancer. Int J Oncol 34: 1069-1075, 2009.

19. Schetter AJ, Leung SY, Sohn JJ, Zanetti KA, Bowman ED, Yanaihara N, Yuen ST, Chan TL, Kwong DL, Au GK, Liu CG, Calin GA, Croce CM and Harris CC: MicroRNA expression profiles associated with prognosis and therapeutic outcome in colon adenocarcinoma. JAMA 299: 425-436, 2008.

20. Slaby O, Svoboda M, Fabian P, Smerdova T, Knoflickova D, Bednarikova M, Nenutil R and Vyzula R: Altered expression of miR-21, miR-31, miR-143 and miR-145 is related to clinicopathologic features of colorectal cancer. Oncology 72: 397-402, 2007.

21. Li X, Zhang Y, Zhang Y, Ding J, Wu K and Fan D: Survival prediction of gastric cancer by a seven-microRNA signature. Gut 59: 579-585, 2010.

22. Dillhoff M, Liu J, Frankel W, Croce C and Bloomston M: MicroRNA-21 is overexpressed in pancreatic cancer and a potential predictor of survival. J Gastrointest Surg 12: 2171-2176, 2008

23. Hwang JH, Voortman J, Giovannetti E, Steinberg SM, Leon LG Kim YT, Funel N, Park JK, Kim MA, Kang GH, Kim SW, Del Chiaro M, et al: Identification of microRNA-21 as a biomarker for chemoresistance and clinical outcome following adjuvant therapy in resectable pancreatic cancer. PLoS One 5: e10630, 2010.
24. He L, Thomson JM, Hemann MT, Hernando-Monge E, Mu D, Goodson S, Powers S, Cordon-Cardo C, Lowe SW, Hannon GJ and Hammond SM: A microRNA polycistron as a potential human oncogene. Nature 435: 828-833, 2005.

25. Volinia S, Calin GA, Liu CG, Ambs S, Cimmino A, Petrocca F, Visone R, Iorio M, Roldo C, Ferracin M, Prueitt RL, Yanaihara N, et al: A microRNA expression signature of human solid tumors defines cancer gene targets. Proc Natl Acad Sci USA 103: 2257-2261, 2006.

26. Wittekind C, Greene FL, Hutter RVP, Klimpfinger $M$ and Sobin LH: TNM Atlas. 5th edition. Springer-Verlag, Berlin, Heidelberg, 2005.

27. Valladares-Ayerbes M, Iglesias-Díaz P, Díaz-Prado S, Ayude D, Medina V, Haz M, Reboredo M, Antolín S, Calvo L and AntónAparicio L: Diagnostic accuracy of small breast epithelial mucin mRNA as a marker for bone marrow micrometastasis in breast cancer: a pilot study. J Cancer Res Clin Oncol 135: 1185-1195, 2009.

28. Borgen E, Naume B, Nesland, et al: Standardization of the immunocytochemical detection of cancer cells in bone marrow and blood. I. Establishment of objective criteria for the evaluation of immunostained cells. Cytotherapy 1: 377-388, 1999.

29. Bustin SA, Benes V, Garson JA, Hellemans J, Huggett J, Kubista M, Mueller R, Nolan T, Pfaffl MW, Shipley GL, Vandesompele J and Wittwer CT: The MIQE guidelines: minimum information for publication of quantitative real-time PCR experiments. Clin Chem 55: 611-622, 2009.

30. Pfaffl MW, Horgan GW and Dempfle L: Relative expression software tool (REST) for group-wise comparison and statistical analysis of relative expression results in real-time PCR. Nucleic Acids Res 30: e36, 2002.

31. Braun S, Vogl FD, Naume B, Janni W, Osborne MP, Coombes RC, Schlimok G, Diel IJ, Gerber B, Gebauer G, Pierga JY, Marth C, et al: A pooled analysis of bone marrow micrometastasis in breast cancer. N Engl J Med 353: 793-802, 2005.

32. Hosch SB, Steffani KD, Scheunemann P and Izbicki JP: Micrometastases from HBP malignancies and metastatic cancer. Hepatob Pancreat Surg 9: 583-591, 2002.

33. Offner S, Schmaus W, Witter K, Baretton GB, Schlimok G, Passlick B, Riethmüller and Pantel K: p53 gene mutations are not required for early dissemination of cancer cells. Proc Natl Acad Sci USA 96: 6942-6946, 1999.

34. Kakeji Y, Maehara Y, Shibahara K, Hasuda S, Tokunaga E, Oki E and Sugimachi K: Clinical significance of micrometastasis in bone marrow of patients with gastric cancer and its relation to angiogenesis. Gastric Cancer 2: 46-51, 1999.

35. Gretschel S, Astrosini Ch, Vieth M, Jöns Th, Tomov T, Höcker M, Schlag PM and Kemmner W: Markers of tumour angiogenesis and tumour cells in bone marrow in gastric cancer patients. Eur J Surg Oncol 34: 642-647, 2008

36. Kaifi JT, Yekebas EF, Schurr P, Obonyo D, Wachowiak R, Busch P, Heinecke A, Pantel K and Izbicki JR: Tumor-cell homing to lymph nodes and bone marrow and CXCR4 expression in esophageal cancer. J Natl Cancer Inst 97: 1840-1847, 2005.

37. Kaifi JT, Reichelt U, Quaas A, Schurr PG, Wachowiak R, Yekebas EF, Strate T, Schneider C, Pantel K, Schachner M, Sauter G and Izbicki JR: L1 is associated with micrometastatic spread and poor outcome in colorectal cancer. Modern Pathol 20: 1183-1190, 2007.

38. Benes V and Castoldi M: Expression profiling of microRNA using real-time quantitative PCR, how to use it and what is available. Methods 50: 244-249, 2010.

39. Navon R, Wang H, Steinfeld I, Tsalenko A, Ben-Dor A and Yakhini Z: Novel rank-based statistical methods reveal microRNAs with differential expression in multiple cancer types. PLoS One 4: e8003, 2009.

40. Haaften G and Agami R: Tumorigenicity of the miR-17-92 cluster distilled. Genes Dev 24: 1-4, 2010.

41. Taguchi A, Yanagisawa K, Tanaka M, Cao K, Matsuyama Y, Goto H and Takahashi T: Identification of hypoxia-inducible factor-1A as a novel target for miR-17-92 microRNA cluster. Cancer Res 68 : 5540-5545, 2008

42. Yan H, Xue G, Mei Q, Wang Y, Ding F, Liu M, Lu M, Tang Y, $\mathrm{Yu} \mathrm{H}$ and Sun S: Repression of the miR-17-92 cluster by $\mathrm{p} 53$ has an important function in hypoxia-induced apoptosis. EMBO J 28: 2719-2732, 2009

43. Dews M, Fox JL, Hultine S, Sundaram P, Wang W, Liu YY, Furth E, Enders GH, El-Deiry W, Schelter JM, Cleary MA and Thomas-Tikhonenko A: The myc-miR-17 92 axis blunts TGF beta signaling and production of multiple TGF beta-dependent antiangiogenic factors. Cancer Res 70: 8233-8246, 2010. 
44. Diaz R, Silva J, Garcia JM, Lorenzo Y, Garcia V, Pena C, Rodriguez R, Munoz C, Garcia F, Bonilla F and Dominguez G: Deregulated expression of miR-106a predicts survival in human colon cancer patients. Genes Chromosomes Cancer 47: 794-802, 2008.

45. Ueda T, Volinia S, Okumura H, Shimizu M, Taccioli C, Rossi S, Alder H, Liu CG, Oue N, Yasui W, Yoshida K, Sasaki H, Nomura S et al: Relation between microRNA expression and progression and prognosis of gastric cancer: a microRNA expression analysis. Lancet Oncol 11: 136-146, 2010.

46. Motoyama K, Inoue H, Mimori K, Tanaka F, Kojima K Uetake H, Sugihara K and Mori M: Clinicopathological and prognostic significance of PDCD4 and microRNA-21 in human gastric cancer. Int J Oncol 36: 1089-1095, 2010.

47. Lagerstedt KK, Kristiansson E, Lönnroth C, Andersson M, Iresjö BM, Gustafsson A, Hansson E, Kressner U, Nordgren S, Enlund $\mathrm{F}$ and Lundholm K: Genes with relevance for early to late progression of colon carcinoma based on combined genomic and transcriptomic information from the same patients. Cancer Informatics 9: 79-91, 2010.
48. Nicole MA, White NMA, Fatoohi E, Metias M, Jung K, Stephan C and Yousef GM: Metastamirs: a stepping stone towards improved cancer management. Nat Rev Clin Oncol 8: 75-84, 2011.

49. Wang P, Zou F, Zhang X, Li H, Dulak A, Tomko RJ, Lazo JS, Wang Z, Zhang L and Yu J: MicroRNA-21 negatively regulates Cdc25A and cell cycle progression in colon cancer cells. Cancer Res 69: 8157-8165, 2009.

50. Nielsen BS, Jørgensen S, Fog JU, Søkilde R, Christensen IJ, Hansen U, Brünner N, Baker A, Møller S and Nielsen HJ: High levels of microRNA-21 in the stroma of colorectal cancers predict short disease-free survival in stage II colon cancer patients. Clin Exp Metastasis 28: 27-38, 2011.

51. Schepeler T, Reinert JT, Ostenfeld MS, Christensen LL, Silahtaroglu AN, Dyrskjot L, Wiuf C, Sorensen FJ, Kruhoffer M, Laurberg S, Kauppinen S, Ørntoft TF and Andersen CL: Diagnostic and prognostic microRNAs in stage II colon cancer. Cancer Res 68: 6416-6424, 2008. 